\title{
Potential therapeutic applications of biosurfactants
}

\author{
Eduardo J. Gudiñaa ${ }^{1 *}$, Vivek Rangarajan ${ }^{2 *}$, Ramkrishna Sen ${ }^{2}$, and Lígia R. Rodrigues ${ }^{1}$ \\ ${ }^{1}$ IBB - Institute for Biotechnology and Bioengineering, Centre of Biological Engineering, University of Minho, Campus de Gualtar, \\ 4710-057, Braga, Portugal \\ ${ }^{2}$ Department of Biotechnology, Indian Institute of Technology (IIT) Kharagpur, West Bengal 721302, India
}

\begin{abstract}
Biosurfactants have recently emerged as promising molecules for their structural novelty, versatility, and diverse properties that are potentially useful for many therapeutic applications. Mainly due to their surface activity, these molecules interact with cell membranes of several organisms and/or with the surrounding environments, and thus can be viewed as potential cancer therapeutics or as constituents of drug delivery systems. Some types of microbial surfactants, such as lipopeptides and glycolipids, have been shown to selectively inhibit the proliferation of cancer cells and to disrupt cell membranes causing their lysis through apoptosis pathways. Moreover, biosurfactants as drug delivery vehicles offer commercially attractive and scientifically novel applications. This review covers the current state-ofthe-art in biosurfactant research for therapeutic purposes, providing new directions towards the discovery and development of molecules with novel structures and diverse functions for advanced applications.
\end{abstract}

\section{Biosurfactants as promising molecules}

Biosurfactants comprise a group of diverse amphipathic molecules with distinct chemical structures produced by several microorganisms. These molecules, which are mainly formed as secondary metabolites, play critical roles in the survival of their producing microorganisms by facilitating nutrient transport, interfering in microbe-host interactions and quorum sensing mechanisms, or by acting as biocide agents [1]. Their recognized potential and biological nature has inspired numerous studies on their possible therapeutic applications (reviewed in $[2,3]$ ). These compounds are superior to synthetic surfactants, owing to their microbial origin, biodegradability, and low toxicity [1]. For that reason they have been widely studied for applications in food and cosmetics industries, enhanced oil recovery, and bioremediation [1]. They are generally classified as low (including glycolipids and lipopeptides) and high molecular weight (polysaccharides, proteins,

\footnotetext{
Corresponding author: Rodrigues, L.R. (lrmr@deb.uminho.pt).

Keywords: biosurfactants; therapeutic applications; antitumor agents; drug delivery systems.

These authors contributed equally to this work.

0165-6147/\$ - see front matter

(C) 2013 Elsevier Ltd. All rights reserved. http://dx.doi.org/10.1016/j.tips.2013.10.002
}

lipoproteins, among others) biosurfactants. Generally, low molecular weight biosurfactants have excellent surface active properties due to their relatively simpler structures. Rhamnolipids (glycolipid) (Figure 1A) and surfactin (lipopeptide) (Figure 1B) are among the best studied biosurfactants.

Biosurfactants can affect the adhesion of microorganisms because they partition at interfaces of fluid phases with distinct polarities and hydrogen bonding [3-5]. Likewise, these compounds can disrupt cell membranes that lead to cell lysis by increased membrane permeability and ultimately to leakage of metabolites [6]. Changes in the physical membrane structure or modifications in protein conformations occur, thus altering significant membrane functions that comprise transport and energy generation [4].

Among all properties of biosurfactants, their antibacterial, antifungal, and antiviral activities, in addition to their anti-adhesive character against pathogens, and probiotic nature, are the most relevant for health-related applications [1-3,7]. Some biosurfactants have been reported as suitable alternatives to synthetic medicines and antimicrobials and may be used as safe and effective therapeutic agents. Their possible applications include gene transfection, as adjuvants for antigens, as inhibitors of fibrin clot formation, as activators of fibrin clot lysis, and also as antiadhesive coatings for biomaterials, incorporated into probiotic preparations to fight urogenital tract infections and for pulmonary immunotherapy [8].

Recently, biosurfactants have been shown to have effects on cancer cells. For instance, the lipopeptide surfactin was found to induce apoptosis in breast cancer cells [9]. Similarly, the glycolipids mannosylerythritol lipids (MELs) and succinoyl trehalose lipids (STLs) have been involved in growth arrest and apoptosis of tumor cells [10-12].

Many other therapeutic applications have been suggested for biosurfactants including novel and attractive uses in nanotechnology mainly based on their flexible selfassembling [13]. For example, a liposome vector containing $\beta$-sitosterol $\beta$-D-glucoside biosurfactant-complexed DNA was successfully used for herpes simplex virus thymidine kinase gene therapy [14]. More recently, nanovectors containing a biosurfactant have been used to increase the efficacy of gene transfection in vitro and in vivo [15].

Nevertheless, although it seems clear that biosurfactants are valuable, multipurpose, and useful molecules for therapeutic uses, some may constitute a risk for humans and should be carefully scrutinized. For example, it is 

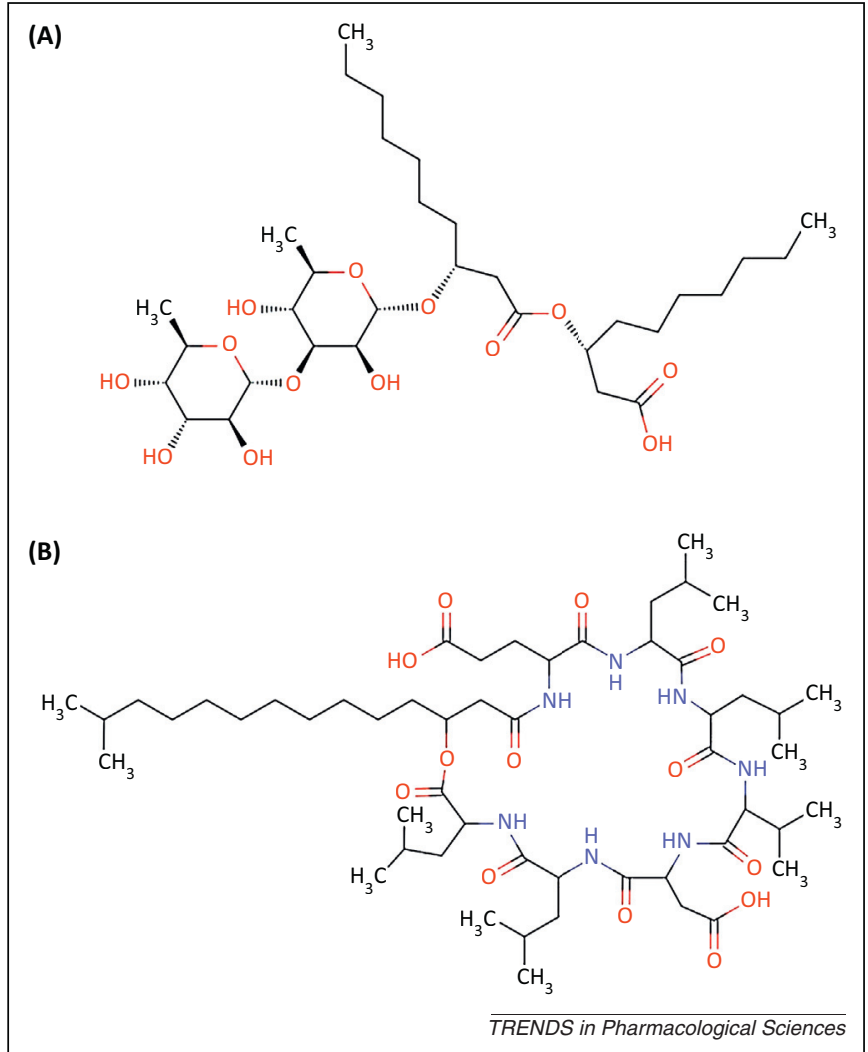

Figure 1. Chemical structure of common biosurfactants: rhamnolipid (A) and surfactin (B).

recognized that Pseudomonas aeruginosa is responsible for severe nosocomial infections, yet this strain produces powerful glycolipids for several medical-related applications $[16,17]$.

This review discusses the current state of biosurfactant research, with an emphasis on potential therapeutic applications. We aim to provide new insights and directions towards discovering molecules with novel structures and diverse functions for cutting edge applications as improved anticancer drugs or nanoscale microemulsion-based drug delivery vectors.

\section{Biosurfactants as antitumor agents}

One of the most exciting findings that has been reported for biosurfactants is their ability to control a variety of mammalian cell functions and therefore their potential to act as antitumor agents interfering with some cancer progression processes (Table 1). Indeed, these molecules have been shown to participate in several intercellular molecular recognition steps such as signal transduction, cell differentiation, and cell immune response, among others [8].

For example, glycolipids (amphipathic molecules consisting of lipids with a carbohydrate attached) have been shown to be involved in growth arrest and apoptosis of mouse malignant melanoma B16 cells. Exposure to increasing concentrations of MELs led to the accumulation of B16 cells in the sub-G0/G1 phase, which is a sign of cells undergoing apoptosis. Furthermore, a sequence of apoptotic events was observed including the condensation of chromatin and DNA fragmentation, thus confirming the apoptosis-inducing potential of MELs in these cells [10].
This report suggests that regulation of the activity of protein kinase $\mathrm{C}$ (PKC) might be associated with apoptosis induced by MELs. Activation of PKC is one of the first events in the signal transduction that leads to a multiplicity of cellular responses. Indeed, members of the PKC family are key factors in cell differentiation, control of growth, and cell death. Additionally, MELs have been shown to induce the differentiation of human promyelocytic leukemia HL60 cells towards granulocytes [18]. MELs were found to markedly increase common differentiation-associated characteristics in granulocytes, such as nitroblue tetrazolium reducing ability, expression of Fc receptors ( $\mathrm{Fc}$ - surface immunoglobulin molecule) and phagocytic activities in HL60 cells. Furthermore, the authors demonstrated that MELs inhibited the activity of PKC in these cells. Their results suggest that the differentiation-inducing activity of MELs might involve changes in membrane-associated molecules. The abovementioned reports indicate that MEL biosurfactants can trigger both apoptotic and differentiation mechanisms $[10,18]$. In other studies, MELs have also shown excellent growth inhibition and differentiation activities against several cancer cell lines [11,19-21]. Likewise, STLs have also been shown to inhibit growth and induce differentiation of human leukemia cells [18,22].

Additionally, sophorolipids have been found to trigger cell differentiation instead of cell proliferation and to inhibit PKC activity in the HL60 human leukemia cell line. This activity is not caused by a simple detergent-like effect but is attributed to a specific interaction with the plasma membrane [12]. The sophorolipid produced by Wickerhamiella domercqiae was shown to induce apoptosis in H7402 human liver cancer cells by blocking the cell cycle at G1 phase, activating caspase-3, and increasing $\mathrm{Ca}^{2+}$ concentration in the cytoplasm [23]. Fu and collaborators [24] investigated the effects of different sophorolipid derivatives against human pancreatic carcinoma cells and demonstrated that the cytotoxic effect was dependent on the derivative (the methyl ester derivative being the most effective), suggesting that distinct mechanisms may be involved in this effect. Similarly, Shao and collaborators [25] investigated the effect of sophorolipid molecules with different structures on human esophageal cancer cell lines. Stronger inhibition was shown for sophorolipids with higher degrees of acetylation, (specifically, $30 \mathrm{mg} / \mathrm{ml}$ of diacetylated lactonic sophorolipid completely inhibited cells), whereas twice the concentration was necessary to obtain the same inhibitory effect with monoacetylated lactonic sophorolipid. The sophorolipid with one double bond in the fatty acid part had the strongest cytotoxic effect, whereas the antitumor activity of acidic sophorolipids was scarce. The authors put forward that different mechanisms may be involved in the anticancer activities observed for the different sophorolipid derivatives [24,25]. Nevertheless, these studies only evaluate the cytotoxicity of these compounds and do not deeply investigate the possible mechanisms underlying such activity. Notwithstanding, because different sophorolipid derivatives were found to exhibit different anticancer activities, these findings suggest that a rational manipulation of the sophorolipid structures, namely with higher acetylation 
Table 1. Biosurfactants with antitumor activity against human cancer cells

\begin{tabular}{|c|c|c|c|c|}
\hline Biosurfactant & Cell line & Description & Activity & Refs \\
\hline $\begin{array}{l}\text { Mannosylerythritol } \\
\text { lipids (MELs) }\end{array}$ & K562 & Myelogenous leukemia & Growth inhibition, differentiation & [11] \\
\hline $\begin{array}{l}\text { Succinoyl trehalose } \\
\text { lipids (STLs) }\end{array}$ & HL60 & Promyelocytic leukemia & Growth inhibition, differentiation & [22] \\
\hline \multirow{3}{*}{ Sophorolipids } & HL60 & Promyelocytic leukemia & Interaction with plasma membrane & [18] \\
\hline & H7402 & Liver cancer & $\begin{array}{l}\text { Growth inhibition, cell cycle arrest, } \\
\text { apoptosis induction }\end{array}$ & [23] \\
\hline & KYSE109/KYSE450 & Esophageal cancer & Growth inhibition & [25] \\
\hline \multirow{4}{*}{$\begin{array}{l}\text { Surfactin or surfactin-like } \\
\text { biosurfactants }\end{array}$} & BEL7402 & Hepatocellular carcinoma & Growth inhibition, apoptosis induction & [32] \\
\hline & K562 & Myelogenous leukemia & $\begin{array}{l}\text { Growth inhibition, cell cycle arrest, } \\
\text { apoptosis induction }\end{array}$ & {$[32,35]$} \\
\hline & LoVo & Colon adenocarcinoma & Growth inhibition, apoptosis induction & [28] \\
\hline & MCF7 & Breast cancer & Growth inhibition, apoptosis induction & {$[9,29,30]$} \\
\hline \multirow[t]{2}{*}{$\varepsilon$-poly-L-lysine } & HeLaS3 & Cervix adenocarcinoma & Growth inhibition & [34] \\
\hline & HepG2 & Hepatocellular liver carcinoma & Growth inhibition & [34] \\
\hline Viscosin & PC3M & Metastatic prostate cancer & Migration inhibition & [36] \\
\hline Serratamolide & BCLL & B-Chronic lymphocytic leukemia & Apoptosis induction & [37] \\
\hline \multirow[t]{2}{*}{ Monoolein } & HeLa & Cervical cancer & Growth inhibition & [39] \\
\hline & U937 & Leukemia cancer & Growth inhibition & [39] \\
\hline $\begin{array}{l}\text { Glycoprotein from } \\
\text { Lactobacillus paracasei }\end{array}$ & T47D/MDA-MB231 & Breast cancer & Growth inhibition, cell cycle arrest & $\begin{array}{l}\text { Unpublished } \\
\text { data }\end{array}$ \\
\hline
\end{tabular}

degree of sophorose, less unsaturation degree of hydroxyl fatty acid, and lactonization, may lead to novel compounds with improved performances.

Other less studied glycolipids have also been reported for their potential antitumor promoting activity, for example, the crude glycolipid from Sphingobacterium detergens against Caco2 human colorectal cancer cells [26].

Lipopeptides, including surfactin, have also been widely studied for their potential antitumor activity against several cancer cell lines [27]. Kim and collaborators [28] showed that surfactin blocks cell proliferation by inducing proapoptotic activity and arresting the cell cycle.

Furthermore, surfactin strongly blocked the PI3K/Akt signaling pathway [PI3K - phosphoinositide 3 kinase; Akt also known as protein kinase $\mathrm{B}$ (PKB) is a serine/threonine-specific protein kinase; both proteins are involved in multiple cellular processes such as cell proliferation and apoptosis]. This pathway is known to play a central role in regulating proapoptotic processes including cell cycle arrest. Altogether, these results suggest that surfactin can downregulate the cell cycle and suppress cancer cell survival. In addition, surfactin purified from the strain Bacillus subtilis CSY191 (probiotic strain) was found to inhibit the growth of MCF7 human breast cancer cells in a dosedependent manner [29]. Cao and collaborators [9] further demonstrated that this lipopeptide induces apoptosis in MCF7 cells through a reactive oxygen species/c-Jun $\mathrm{N}$-terminal kinase (ROS/JNK)-mediated mitochondrial/ caspase pathway. In addition, the authors showed that surfactin induces ROS formation, leading to mitochondrial permeability and membrane potential collapse that ultimately results in an increase of ion calcium concentration in the cytoplasm [30]. Afterwards, cytochrome c released from mitochondria to the cytoplasm activates caspase-9, eventually inducing apoptosis. Moreover, surfactin was shown to inhibit the proliferation of MCF7 cells through cell arrest at the G2/M phase [31]. Indeed, surfactin induced accumulation of the tumor suppressor p53 and cyclin kinase inhibitor p21 $1^{\text {wafl/cip1 }}$, and inhibited the activity of the G2specific kinase, cyclin $\mathrm{B} 1 / \mathrm{p} 34^{\mathrm{cdc} 2}$. These findings suggest that surfactin caused the G2/M arrest of MCF7 cells through the regulation of their cell cycle factors. The same research group demonstrated the cytotoxic effect of surfactin, in a dose-dependent manner, against the human chronic myelogenous leukemia cells K562 and the hepatic carcinoma cells BEL7402 [32]. Wang et al. [33] also demonstrated that surfactin induces apoptosis in HepG2 cells through ROSendoplasmic reticulum stress (ERS) $-\mathrm{Ca}^{2+}$-extracellular signal-regulated protein kinase (ERK) pathways. A summary of the proposed mechanisms underlying the anticancer effect of surfactin is illustrated in Figure 2.

Different lipopeptides produced by Bacillus, Pseudomonas, and Serratia species have also exhibited antitumoral activity against various human cancer cells [27,34-38].

Because there is an enormous diversity of microbial surfactants, new molecules with interesting antitumor activities are continuously being reported. This is the case of the biosurfactant monoolein produced by the dematiaceous fungus Exophiala dermatitidis SK80 [39]. The biosurfactant effectively inhibited the proliferation of cervical cancer (HeLa) and leukemia (U937) cell lines in a dosedependent manner. Interestingly, no cytotoxicity was found with normal cells, even when high concentrations were used. Cell and DNA morphological changes observed 


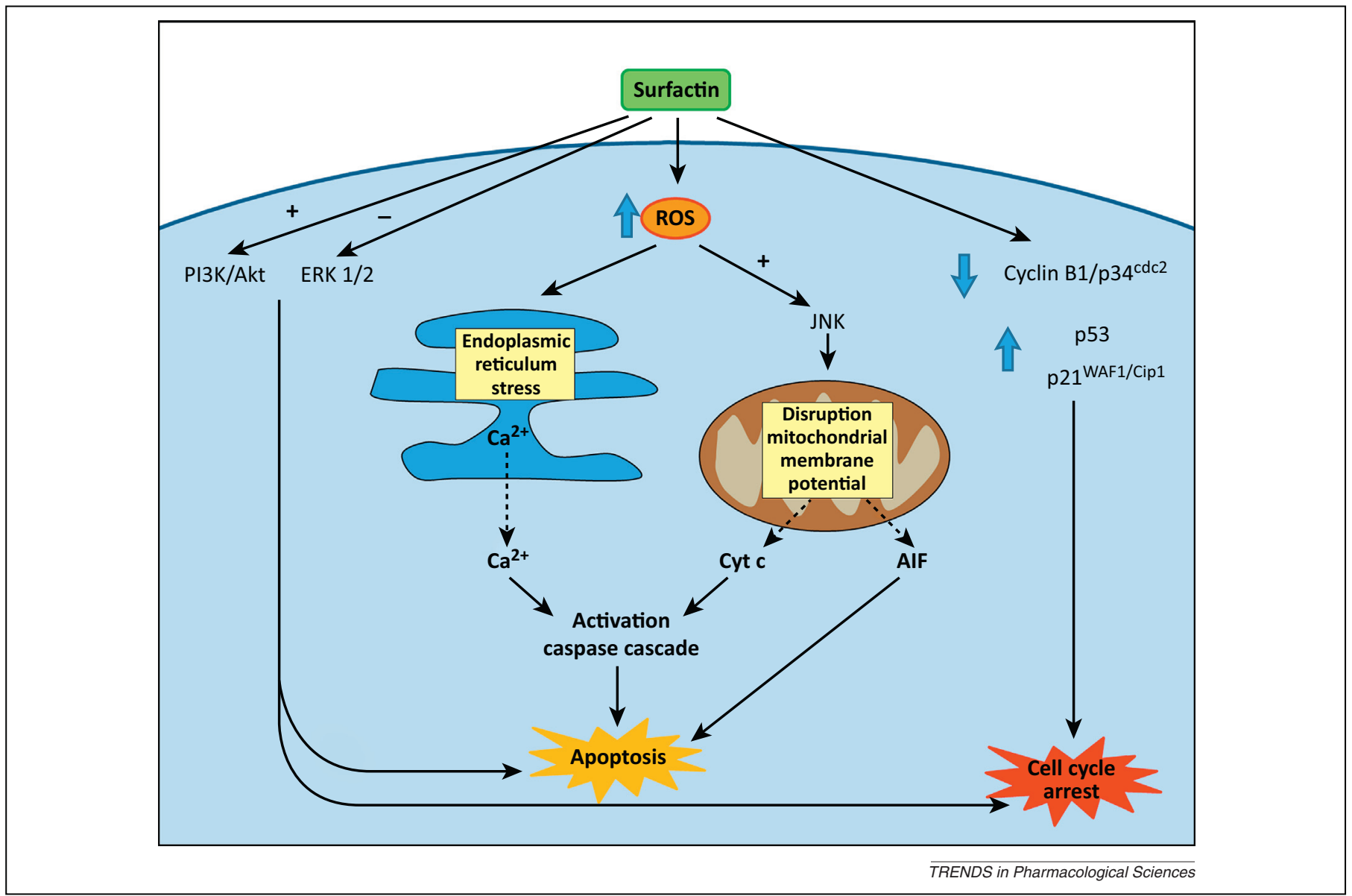

Figure 2. Proposed mechanisms involved in the antitumoral activity of surfactin. Abbreviations: AlF, apoptosis-inducing factor; Cyt c, cytochrome c; ERK, extracellular signal-regulated protein kinase; JNK, c-Jun N-terminal kinase; PI3K, phosphoinositide 3-kinase; ROS, reactive oxygen species.

in both cancer cell lines include cell shrinkage, membrane blebbing, and DNA fragmentation. Another example is the glycoprotein produced by Lactobacillus paracasei [40] that is active against human breast cancer cells.

Although researchers have been reporting the antitumor potential of biosurfactants, few studies have investigated the mechanisms involved in such activity. Indeed, most studies use a single cancer cell line to assess the cytotoxicity of the biosurfactants without using proper controls (e.g., normal cell lines), which means that these compounds may not be as specific as desired, and by contrast may be only effective against that single cell line. Also, some of these studies are somewhat preliminary and support their conclusions in a simple assessment of cell viability using a single method. Furthermore, although some results seem very promising, they are based on cell lines and further in vivo experiments must be conducted in order to validate the potential of the compounds. The shortcoming of most studies on the anticancer potential of biosurfactants is undoubtedly the lack of detail concerning the mechanisms that underlie the activity of these compounds.

Biosurfactants have been implicated in several intercellular molecular recognition steps through interference with specific molecules. For example, it has been reported that the profiles of lipids in normal and cancerous tissues differ, such as the cell membrane lipids [41-45]. Because lipids are surface active, any change in lipid profiles can lead to altered surface activity profiles. These membrane lipids can, however, interact with biosurfactants. The interaction between these two surface active molecules can lead to important cell membrane modifications and ultimately to cell death. Lipid composition determines the structure, function, and integrity of biological membranes, and phosphatidylcholine (PC) and sphingomyelin (SM), in particular, play a role in stabilizing the bilayer structure. Preetha and collaborators [41] demonstrated that the phospholipid profiles of normal and cancerous cervical tissues were significantly different, namely, that the PC levels in cancer tissue were nearly 5 -fold higher than those in normal tissue. PC and SM were found to be the major phospholipid components in cancerous and normal cervical tissues, respectively. The authors suggest that changes in membrane fluidity, due to PC and SM levels (these phospholipids confer rigidity to the membrane), in turn might affect the permeability of the cancer cell membranes. Therefore, new therapeutic strategies may be designed, considering that the use of biosurfactants can alter lipid content (specifically PC and SM), to fluidize rigid cancerous tissues and to modulate interfacial properties. The increased rigidity manifests as a lower surface tension and can reduce the penetration of drugs through such membranes. The role of fluidizers in reversing these rigidifying effects and improving drug penetration in cancerous 
tissues has been postulated. Furthermore, these findings open interesting perspectives for the development of drug carriers based on surface active molecules, such as biosurfactants, that can interact with cell membrane lipids, or for the design of agents that can interact with other lipids involved in several cellular processes. For instance, biosurfactants could be used to influence the activity of sphingolipids, because these lipids emerged as effector molecules, which control various aspects of cell growth, proliferation, and anticancer therapeutics [46].

Finally, the ability of biosurfactants to disrupt cell membranes, leading to a sequence of events that include lysis, increased membrane permeability, and metabolite leakage, has also been suggested as a probable mechanism of antitumor activity [47].

\section{Biosurfactants as drug delivery agents}

The discovery of new drugs and novel drug delivery systems (DDSs) with improved efficacies has made a significant impact on our ability to treat many types of diseases [48]. Ideally, a controlled DDS should hold two important characteristics: (i) optimal drug loading capacity, which leads to increased bioavailability of drug and ability to reach the target of interest, and (ii) the subsequent release of the drug in a controlled and phased manner. To accomplish these two essential purposes, different types of pharmaceutical carriers such as polymeric, particulate, macromolecular, and cellular carriers have been tested and are currently used. Of these, the particulate type exists in a dispersed colloidal form with structures that include microspheres, nanoparticles, lipid particles, micelles, and vesicular systems such as liposomes, noisome, virosomes, and sphingosomes [49]. Microemulsions have emerged as novel DDSs suitable for transdermal, topical, oral, nasal, ocular, intravenous, parenteral, and other routes of drug administration (reviewed in [48]). They have been the focus of significant attention of researchers owing to their easiness in formulation. However, systematic and preclinical studies are required before an optimal formulation can guarantee the safety and efficacy criteria for a given route of drug administration. Because microemulsion systems are thermodynamically stable [50], more caution has been particularly used in the formulation of self-microemulsifying drug delivery systems (SMEDDSs) for oral or parenteral routes. It is important to note that most DDSs fail when these routes of administration are used [51]. Several reasons have been reported for DDS failure, ranging from poor efficacy in delivering the drug to the drug precipitation due to dilution by biological fluids before reaching the target site. However, the important criterion that most formulations fail to abide is the judicious use of biocompatible and biodegradable pharmaceutical agents as their ingredients.

\section{Microemulsion}

A microemulsion-based colloidal DDS generally comprises an aqueous phase (usually water), an oil phase, a surfactant, and often includes a co-surfactant or co-solvent. The surfactant, which is the principal ingredient of a microemulsion system, self-aggregates to form templates of varying structures. These structures can encapsulate/solubilize a hydrophobic or hydrophilic drug in the presence of a dispersed phase [oil in the case of oil-in-water $(\mathrm{O} / \mathrm{W})$ and water in the case of water-in-oil (W/O) microemulsions] within its structural core (spherical in most cases), thereby partitioning the dispersed phase from the continuous phase [52]. Typically, a global microemulsion system exhibits a wide range of structures of distinct nanometric-scaled geometries (e.g., worm-like, bicontinuous sponge-like, liquid crystalline, or hexagonal, spherical swollen micelles) involving the formation of one, two, or even three phases. Furthermore, the thermodynamic dependency of these microemulsion systems clearly suggests that any change in the composition and temperature of the system will cause phase separation and lead to eventual loss of the emulsified drug.

\section{Green molecules in drug formulation}

In recent years, formulators have been actively seeking pharmaceutically acceptable excipients to design safer microemulsions. Previous efforts have typically used synthetic hydrocarbon oils such as heptanes, dodecane, and cyclic oils such as cyclohexane, and surfactants with 12 carbon hydrophobic chains such as sodium dodecyl sulfate and tetraethylene glycol monododecyl ether, which are not approved for use in pharmaceutical formulations, and can present biocompatibility issues and exhibit some toxic effects [53]. To a reasonable extent, biocompatibility has been guaranteed through the use of lecithins and non-ionic surfactants such as Brijs, Arlacel 186, Spans, Tweens, and AOT, which are amphiphiles (components of microemulsion systems) that have been widely demonstrated to exhibit a high biocompatibility [54]. At the same time, it is encouraging to see a recent tendency to use natural oils as alternatives for synthetic oils and surfactants to formulate nontoxic pharmaceutically acceptable microemulsion systems. Vegetable oils have been the focus of huge interest, but it is relatively difficult to solubilize them in microemulsions [55]. Similarly, natural surfactants have emerged as potential alternatives for their synthetic counterparts. In particular, non-ionic surfactants such as sucrose esters, containing a hydrophilic sucrose group and fatty acid chains of varying degrees as a lipophilic group, have been widely employed in microemulsion formulation [56,57].

Biosurfactants have emerged as a better alternative to their synthetic counterparts. The recent trend in their use as templates for nanoparticle synthesis indicates the constantly increasing potential of biosurfactants to serve as greener alternatives to their synthetic counterparts [58].

\section{Challenges, selection guidelines, and future prospects of biosurfactants in drug delivery applications}

Although it is very difficult to predict the nature and stability of a microemulsion-based DDS, data reported in the literature can drive the selection of the most appropriate oil/biosurfactant system. Environmental conditions can strongly affect some biosurfactants and consequently their self-assembly. Regarding rhamnolipids and surfactin, adequate results are available pertaining to their structural aspects at different interfaces and solutions, as described below [59-62]. However, these molecules, 
unlike synthetic surfactants, have relatively vague demarcations between their hydrophilic and lipophilic groups. The very complex nature of the head groups (e.g., amino acids in lipopeptide and saccharides in glycolipids) further complicates proper assessment of their structure, because they can adopt varied structures with only a slight change in the environment. This behavior is linked to the presence of one and two carboxylic acids in di-rhamnolipids and surfactin, respectively.

Generally, it can be assumed that these biosurfactants are anionic at high $\mathrm{pH}$ values (due to the presence of carboxylic groups) and non-ionic at low $\mathrm{pH}$ values [63]. Also, structure transition from micellar to lamellar upon electrolyte addition has been reported $[64,65]$. Such possibility of manipulating structure transition may be of great interest to tailor a DDS for a given drug, or to confer its functionality in particular environmental conditions (controlled drug release), for example, triggered by $\mathrm{pH}$, temperature, or salt concentrations [66]. Aggregation of surfactants in a lamellar arrangement can occur if one of the following requirements is met. High surfactant concentrations in water often lead to a lyotropic lamellar liquid crystalline phase. Double-tailed amphiphiles commonly form bilayer sheets, because their most hydrated state enables the molecules to pack only in a lamellar arrangement. Upon closing, these sheets form vesicles. Lamellar aggregates can also be formed from mixtures of anionic and cationic surfactants in water, mixtures of ionic surfactants and long-chain alcohols in water, or electrolyte solution. As previously mentioned, some surfactant molecules in aqueous solution are spontaneously transformed from micelles into a lamellar arrangement in the presence of a high salt concentration. This change in aggregate morphology is facilitated by an increase in counter ion binding and dehydration of the surfactant head groups and bound counter ions. On a larger scale, interactions between lamellae occur, leading to the formation of either unilamellar vesicles or multilayered systems. Owing to the lack of systematic studies on the characteristics of biosurfactant microemulsion systems, such as phase behavior and its stability under different physicochemical conditions and compatibility of oil and co-surfactants, drug delivery applications of biosurfactants remain to be developed. However, it is strongly believed that prior knowledge about the characteristics of the system and its components through a proper assessment of various parameters such as the hydrophilic-lypophilic balance (HLB), critical packing parameter $(\mathrm{CPP})$, and Winsor $\mathrm{R}$ ratio would significantly reduce the complexity of a rational choice of components that ultimately lead to a successful microemulsion formulation. Furthermore, these parameters can guide researchers in designing more realistic drug formulations for a specific route of administration. The HLB affects the stability of the emulsion and represents the relative contribution of hydrophilic and lipophilic groups of the surfactant. As a general rule, low HLB values (3-6) favor the formation of W/O microemulsions, whereas high HLB values $(8-18)$ favor O/W microemulsions. For surfactants with very high HLB values $(>20)$, often a cosurfactant is required to reduce their effective HLB value. Amphiphilic molecules exhibit a broad and puzzling phase

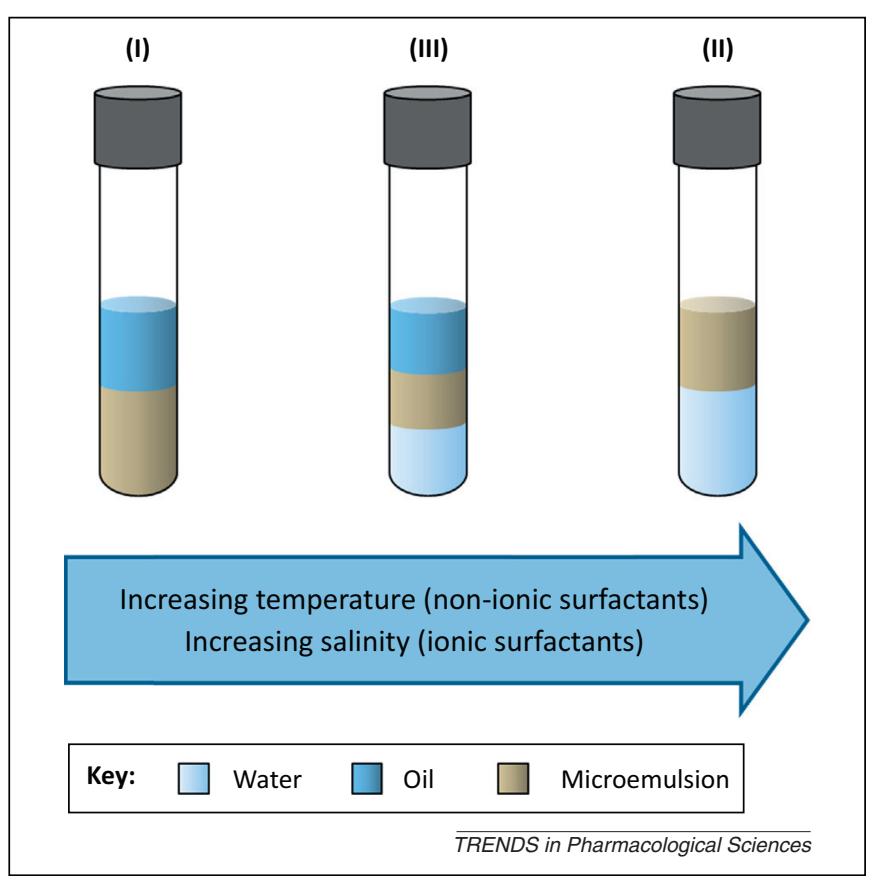

Figure 3. Winsor classification of microemulsions. Microemulsions can exist in three forms, known as Winsor type microemulsions. Type I (O/W), when the watersurfactant interaction is stronger than oil-surfactant interaction $(R<1)$, microemulsions solubilize oil in spherical normal micelles within the watercontinuous phase. Type II (W/O), when the strength of oil-surfactant interaction is stronger than water-surfactant interaction $(R>1)$, microemulsions solubilize water in reverse micelles within the oil-continuous phase. Type III, when the interactions are balanced $(R=1)$; in this case, microemulsions are three-phase systems in which the middle phase microemulsions are in equilibrium with both excess oil and excess water phases.

behavior and offer interesting technical challenges regarding their application in DDSs. These molecules spontaneously self-assemble into a wide variety of structures, including spherical (surfactin) and cylindrical (rhamnolipids) micelles, which depend on several environmental conditions. The CPP provides an idea on the ability of a surfactant to form aggregates corresponding to their own geometries. The Winsor $\mathrm{R}$ parameter represents the ratio of the total interaction energies (per unit area of interface) of the surfactant for the $\mathrm{O}$ and $\mathrm{W}$ phases and is also dependent on environmental factors (Figure 3).

Some of the most relevant properties of surfactin and di-rhamnolipids for the formulation of microemulsions are summarized in Table 2. The HLB and CPP values suggest that these two biosurfactants are able to form $\mathrm{O} / \mathrm{W}$ microemulsions, provided that alkaline conditions are maintained. High surface activities imply that only relatively low quantities of these biosurfactants are required to formulate a microemulsion for drug delivery. Furthermore, the high HLB value in the case of rhamnolipids indicates that a co-surfactant may often be required to alter the HLB value leading to microemulsion formation. Xie et al. [63] studied the effect of different alcohols (three to eight carbons) as co-surfactants on the microemulsion phase behavior (rhamnolipid/n-heptane/water system). Addition of alkali favorably changed the hydrophobic nature of rhamnolipids at low $\mathrm{pH}$ to a hydrophilic anionic surfactant. This study showed that excess of sodium ions influenced the phase behavior of the system by minimizing 
Table 2. Important properties guiding biosurfactant selection for microemulsion formulation

\begin{tabular}{|c|c|c|c|c|c|c|}
\hline \multirow[t]{2}{*}{ Biosurfactant } & \multirow{2}{*}{$\begin{array}{l}\text { cmc }(\mu \mathrm{M}) / \text { surface } \\
\text { tension }(\mathrm{mN} / \mathrm{m})\end{array}$} & \multirow[t]{2}{*}{$M_{w}(\mathrm{Da})$} & \multirow{2}{*}{\begin{tabular}{|l|} 
HLB \\
Experimental $^{\mathbf{a}}$
\end{tabular}} & \multicolumn{2}{|l|}{ CPP $b$} & \multirow[t]{2}{*}{ Comments } \\
\hline & & & & Empirical & Experimental & \\
\hline Surfactin & $20 / 27.2$ & 1035 & $10-12$ & 0.1435 & - & $\begin{array}{l}\text { HLB indicates favoring } \mathrm{O} / \mathrm{W} \text { microemulsion } \\
\mathrm{CPP}(<1 / 3) \text { indicates favoring spherical } \\
\text { micelles }\end{array}$ \\
\hline Di-rhamnolipid & $110 / 29.0$ & 650 & $22-24$ & 0.38 & 0.5 & $\begin{array}{l}\text { HLB indicates favoring } \mathrm{O} / \mathrm{W} \text { microemulsion } \\
\text { CPP }(<0.5) \text { indicates favoring mesophase } \\
\text { of cylinders }\end{array}$ \\
\hline
\end{tabular}

${ }^{a}$ For surfactin, it is only a suggestive value based on its ability to reduce surface tension to $27.2 \mathrm{mN} / \mathrm{m}$.

${ }^{b}$ Area for empirical CPP calculation is considered as $147 \AA^{2}$ for surfactin [62] and $80 \AA^{2}$ for rhamnolipid [59].

electrostatic repulsions between the charged head groups. Because the rhamnolipid displayed strong affinity towards water, only Winsor type I microemulsion could be obtained. Moreover, it was reported that increasing the chain length of the alcohol reduced the phase existence area of a twophase microemulsion system, and also that the phase existence region of the single phase microemulsion was maximum for n-butanol. These results suggest that biosurfactants are suitable for emergent drug delivery applications. Nguyen and Sabatini [67] used rhamnolipids as a co-surfactant to alter the HLB value of methyl ester ethoxylate, a biorenewable surfactant, to form a microemulsion with the limonene O/W system, and oleyl alcohol was used as a hydrophilic linker.

Recently, Onaizi et al. [68] studied the micellization and interfacial behavior of a mixture of surfactin and sodium dodecylbenzylsulfonate, showing that the formation of mixed micelles was thermodynamically feasible. Owing to the previously mentioned biosurfactant features, mixed biosurfactant-synthetic surfactant systems are advantageous because they represent greener and sustainable formulations compared with conventional systems. Furthermore, these mixed systems may reduce the costs associated with the exclusive use of biosurfactants and could also encourage the development of a more efficient mixture.

In a separate study, the glycolipid MEL-A synthesized by Pseudozyma antarctica was used to investigate the phase behavior of a ternary MEL-A/water/n-decane system [69]. The MEL-A-stabilized system formed a W/O microemulsion without the need for co-surfactants. Nguyen et al. [70] successfully formulated the most broadly studied biocompatible lecithin-based microemulsion in combination with rhamnolipid and sophorolipid biosurfactants. These microemulsions showed remarkable stability for temperatures up to $40^{\circ} \mathrm{C}$ and an electrolyte concentration of $4 \%$ $(\mathrm{w} / \mathrm{v})$, thus making them suitable for cosmetic and drug delivery applications. Taking a cue from these developments, microemulsions can be designed based on a cautious combination of an oil phase and a suitable biosurfactant.

Biosurfactants, in addition to their imminent potential for application in microemulsion-based drug formulations, have also been reported for use in triggered and targeted drug delivery. Shim et al. [71] successfully demonstrated the enhanced delivery of small interfering RNA (siRNA) in HeLa cells using cationic surfactin liposomes when compared with surfactin-free liposomes. This study also indicated that surfactin-containing liposomes with their higher biocompatibility may improve the specific silencing of the gene of interest, that is, a more efficient delivery system led to an increase of the cellular uptake of siRNA, thus increasing the specific knockdown effect. In a recent study, Cheow and Hadinoto [72] evaluated the rhamnolipids from $P$. aeruginosa biofilm for their ability to trigger the release of a drug encapsulated in lipid-polymer coated hybrid nanoparticles. In such a way, it was possible to trigger the drug release in the vicinity of the $P$. aeruginosa colonies, thus improving the antibacterial effectiveness of those nanoparticles. Although these studies were aimed for rhamnolipid triggered drug release by $P$. aeruginosa biofilm cultures embedded in expectoration under in vivo conditions, the concept of targeted and triggered drug release using biosurfactants can be further applied to the development of DDSs.

\section{Concluding remarks}

Lipopeptide, glycolipid, and other types of biosurfactants, owing to their structural novelty and diverse biophysical properties, have recently emerged as possible broadspectrum agents for cancer chemotherapy/biotherapy and as safe vehicles or ingredients in drug delivery formulations. Many new applications of these biomolecules have been suggested, mainly owing to their significant surface active properties that enable them to interact with cell membranes or surrounding environments to bring about the desired effect as a therapeutic molecule or as a part of a DDS. The search for safe and biocompatible biosurfactants for such applications will drive this field of research in the coming years. The abilities of these molecules to selectively inhibit the proliferation of cancer cell lines and disrupt cell membranes causing cellular lysis by operating apoptotic machineries may provide the clues for the mechanism and mode of their actions. A better understanding of the underpinning principles vis-à-vis the mechanisms of actions at the molecular level would prompt researchers to develop a blueprint of the internal proceedings that can guide in conducting preclinical studies and clinical trials at a later stage.

Drug delivery is another promising area wherein biosurfactants can find potential therapeutic application. Despite the ever increasing demand of biosurfactants for commercial applications, their use in drug delivery requires further research on the interactions between the different components in microemulsions. Limited reports addressing safety issues on the use of biosurfactants as adjuvants in microemulsion formulations are available. Moreover, hemolytic activity of most of the 
reported biosurfactants and the scarcity of clinical data on the use and validation of such molecules in animal models and human volunteers pose a major challenge in preparing safe drug delivery formulations. Nevertheless, some biosurfactants have proven their efficacy in cosmetic and antibiotic formulations and additionally fulfilled the requirements of the drug regulatory bodies worldwide for biocompatible and nontoxic excipients, thus paving the way for the successful implementation of these molecules in drug delivery formulations.

\section{Acknowledgments}

The authors acknowledge Fundação para a Ciência e a Tecnologia (FCT) (Portugal) and the Department of Science and Technology (DST) (India) for financial support of the project 'MEDSURF - The potential use of biosurfactants for medical applications' developed under the scope of a bilateral agreement between Portugal and India.

\section{References}

1 Marchant, R. and Banat, I.M. (2012) Microbial biosurfactants: challenges and opportunities for future exploitation. Trends Biotechnol. 30, 558-565

2 Fracchia, L. et al. (2012) Biosurfactants and bioemulsifiers biomedical and related applications: present status and future potentials. In Biomedical Science, Engineering and Technology (Ghista, D.N., ed.), pp. 325-370, InTech

3 Rodrigues, L.R. (2011) Inhibition of bacterial adhesion on medical devices. In Bacterial Adhesion: Biology, Chemistry, and Physics, Series: Advances in Experimental Medicine and Biology (Linke, D. and Goldman, A., eds), pp. 351-367, Springer

4 Van Hamme, J.D. et al. (2006) Physiological aspects. Part 1 in a series of papers devoted to surfactants in microbiology and biotechnology. Biotechnol. Adv. 24, 604-620

5 Sodagari, M. et al. (2013) Effect of rhamnolipids on initial attachment of bacteria on glass and octadecyltrichlorosilane-modified glass. Colloids Surf. B: Biointerfaces 103, 121-128

6 Bharali, P. et al. (2013) Rhamnolipid (RL) from Pseudomonas aeruginosa OBP1: a novel chemotaxis and antibacterial agent. Colloids Surf. B: Biointerfaces 103, 502-509

7 Raaijmakers, J.M. et al. (2010) Natural functions of lipopeptides from Bacillus and Pseudomonas: more than surfactants and antibiotics. FEMS Microbiol. Rev. 34, 1037-1062

8 Rodrigues, L.R. et al. (2006) Biosurfactants: potential applications in medicine. J. Antimicrob. Chemother. 57, 609-618

9 Cao, X.H. et al. (2010) Surfactin induces apoptosis in human breast cancer MCF-7 cells through a ROS/JNK-mediated mitochondrial/ caspase pathway. Chem. Biol. Interact. 183, 357-362

10 Zhao, X. et al. (2000) Treatment of mouse melanoma cells with phorbol 12-myristate 13-acetate counteracts mannosylerythritol lipid-induced growth arrest and apoptosis. Cytotechnology 33, 123-130

11 Isoda, H. and Nakahara, T. (1997) Mannosylerythritol lipid induces granulocytic differentiation and inhibits the tyrosine phosphorylation of human myelogenous leukemia cell line K562. Cytotechnology 25, 191-195

12 Isoda, H. et al. (1995) Succinoyl trehalose lipid induced differentiation of human monocytoid leukemic cell line U937 into monocytemacrophages. Cytotechnology 19, 79-88

13 Kitamoto, D. et al. (2009) Self-assembling properties of glycolipid biosurfactants and their potential applications. Curr. Opin. Colloid Interface Sci. 14, 315-328

14 Maitani, Y. et al. (2006) Liposome vector containing biosurfactantcomplexed DNA as herpes simplex virus thymidine kinase gene delivery system. J. Liposome Res. 16, 359-372

15 Nakanishi, M. et al. (2009) Nano vectors with a biosurfactant for gene transfection and drug delivery. J. Drug Deliv. Sci. Technol. 19, $165-169$

16 Hosková, M. et al. (2013) Characterization of rhamnolipids produced by non-pathogenic Acinetobacter and Enterobacter bacteria. Bioresour. Technol. 130, 510-516
17 Abbasi, H. et al. (2013) Interaction of a bacterial monorhamnolipid secreted by Pseudomonas aeruginosa MA01 with phosphatidylcholine model membranes. Chem. Phys. Lipids 165, 745-752

18 Isoda, H. et al. (1997) Microbial extracellular glycolipid induction of differentiation and inhibition of protein kinase $\mathrm{C}$ activity of human promyelocytic leukaemia cell line HL60. Biosci. Biotechnol. Biochem. $61,609-614$

19 Wakamatsu, Y. et al. (2001) Mannosylerythritol lipid induces characteristics of neuronal differentiation in PC12 cells through an ERK related signal cascade. Eur. J. Biochem. 268, 374-383

20 Isoda, H. et al. (1997) Differentiation of human promyelocytic leukemia cell line HL60 by microbial extracellular glycolipids. Lipids 32, 263-271

21 Recke, V.K. et al. (2013) Lipase-catalyzed acylation of microbial mannosylerythritol lipids (biosurfactants) and their characterization. Carbohydr. Res. 373, 82-88

22 Sudo, T. et al. (2000) Induction of the differentiation of human HL-60 promyelocytic leukemia cell line by succinoyl trehalose lipids. Cytotechnology 33, 259-264

23 Chen, J. et al. (2006) Sophorolipid produced from the new yeast strain Wickerhamiella domercqiae induces apoptosis in H7402 human liver cancer cells. Appl. Microbiol. Biotechnol. 72, 52-59

$24 \mathrm{Fu}$, S.L. et al. (2008) Sophorolipids and their derivatives are lethal against human pancreatic cancer cells. J. Surg. Res. 148, 77-82

25 Shao, L. et al. (2012) Bioactivities of sophorolipid with different structures against human esophageal cancer cells. J. Surg. Res. 173, 286-291

26 Burgos-Díaz, C. et al. (2013) In vitro study of the cytotoxicity and antiproliferative effects of surfactants produced by Sphingobacterium detergens. Int. J. Pharm. 453, 433-440

27 Sivapathasekaran, C. et al. (2010) Marine bacterium derived lipopeptides: characterization and cytotoxic activity against cancer cell lines. Int. J. Pept. Res. Ther. 16, 215-222

28 Kim, S.Y. et al. (2007) Surfactin from Bacillus subtilis displays antiproliferative effect via apoptosis induction, cell cycle arrest and survival signaling suppression. FEBS Lett. 581, 865-871

29 Lee, J.H. et al. (2012) The production of surfactin during the fermentation of Cheonggukjang by potential probiotic Bacillus subtilis CSY191 and the resultant growth suppression of MCF-7 human breast cancer cells. Food Chem. 131, 1347-1354

30 Cao, X.H. et al. (2011) ROS-Ca ${ }^{2+}$ is associated with mitochondria permeability transition pore involved in surfactin-induced MCF-7 cells apoptosis. Chem. Biol. Interact. 190, 16-27

31 Cao, X.H. et al. (2009) Evaluation of a lipopeptide biosurfactant from Bacillus natto Tk-1 as a potential source of anti-adhesive, antimicrobial and antitumor activities. Braz. J. Microbiol. 40, 373-379

32 Cao, X.H. et al. (2009) Surfactin induces apoptosis and G2/M arrest in human breast cancer MCF-7 cells through cell cycle factor regulation. Cell Biochem. Biophys. 55, 163-171

33 Wang, C. et al. (2013) Surfactin-induced apoptosis through ROS-ERS$\mathrm{Ca}^{2+}$-ERK pathways in HepG2 cells. Cell Biochem. Biophys. http:// dx.doi.org/10.1007/s12013-013-9676-7

34 El-Sersy, N.A. et al. (2012) Antibacterial and anticancer activity of $\varepsilon$-poly-L-lysine ( $\varepsilon-\mathrm{PL}$ ) produced by a marine Bacillus subtilis sp. J. Basic Microbiol. 52, 513-522

35 Wang, C.L. et al. (2007) Induction of apoptosis in human leukemia K562 cells by cyclic lipopeptide from Bacillus subtilis natto T-2. Peptide $28,1344-1350$

36 Saini, H.S. et al. (2008) Efficient purification of the biosurfactants viscosin from Pseudomonas libanensis strain M9-3 and its physicochemical and biological properties. J. Nat. Prod. 71, 1011-1015

37 Escobar-Diaz, E. et al. (2005) AT514, a cyclic depsipeptide from Serratia marcescens, induces apoptosis of B chronic lymphocytic leukemia cells: interference with the Akt/NF $\mathrm{kB}$ survival pathway. Leukemia 19, 572-579

38 Seo, H.R. et al. (2009) Identification of Bacillus cereus in a Chungkukjang that showed high anticancer effects against AGS human gastric adenocarcinoma cells. J. Med. Food 12, $1274-1280$

39 Chiewpattanakul, P. et al. (2010) Bioproduction and anticancer activity of biosurfactant produced by the dematiaceous fungus Exophiala dermatitidis SK80. J. Microbiol. Biotechnol. 20, 1664-1671 
40 Gudiña, E.J. et al. (2010) Isolation and functional characterization of a biosurfactant produced by Lactobacillus paracasei. Colloids Surf. B: Biointerfaces 76, 298-304

41 Preetha, A. et al. (2005) Surface activity, lipid profiles and their implications in cervical cancer. J. Cancer Res. Ther. 1, 180-186

42 Reynier, M. et al. (1991) Differences in lipid characteristics of undifferentiated and entercytic differentiated HT29 human colonic cells. Cancer Res. 51, 1270-1277

43 Hilvo, M. et al. (2011) Novel theranostic opportunities offered by characterization of altered membrane lipid metabolism in breast cancer progression. Cancer Res. 71, 3236-3245

44 Bathen, T.F. et al. (2000) Analysis and classification of proton NMR spectra of lipoprotein fractions from healthy volunteers and patients with cancer or CHD. Anticancer Res. 20, 2393-2408

45 Griffin, J.L. and Shockor, J.P. (2004) Metabolic profiles of cancer cells. Nat. Rev. Cancer 4, 551-561

46 Saddoughi, S.A. et al. (2008) Roles of bioactive sphingolipids in cancer biology and therapeutics. Subcell. Biochem. 49, 413-440

47 Janek, T. et al. (2013) Lipopeptide biosurfactant pseudofactin II induced apoptosis of melanoma A375 cells by specific interaction with the plasma membrane. PLoS ONE 8, e57991

48 Fanun, M. (2012) Microemulsions as delivery systems. Curr. Opin. Colloid Interface Sci. 17, 306-313

49 Gangwar, M. et al. (2012) Recent advances in various emerging vesicular systems: an overview. Asian Pac. J. Trop. Biomed. 2, S1176-S1188

50 Anton, N. and Vandamme, T.F. (2011) Nano-emulsions and microemulsions: clarifications of the critical differences. Pharm. Res. 28, 978-985

51 Crommelin, D.J.A. and Florence, A.T. (2013) Towards more effective advanced drug delivery systems. Int. J. Pharm. 454, 496-511

52 Israelachvili, J. (1994) The science and applications of emulsions - an overview. Colloids Surf. A 91, 1-8

53 Lawrence, M.J. and Rees, G.D. (2012) Microemulsion-based media as novel drug delivery systems. Adv. Drug Deliv. Rev. 64, 175-193

54 Paul, B.K. and Moulik, S.P. (2001) Uses and applications of microemulsions. Curr. Sci. 80, 990-1001

55 Do, L.D. et al. (2009) Environmentally friendly vegetable oil microemulsions using extended surfactants and linkers. J. Surfact. Deterg. 12, 91-99

56 Chansanroj, K. and Betz, G. (2010) Sucrose esters with various hydrophilic-lipophilic properties: novel controlled release agents for oral drug delivery matrix tablets prepared by direct compaction. Acta Biomater. 6, 3101-3109
57 Csizmazia, E. et al. (2012) Ibuprofen penetration enhance by sucrose ester examined by ATR-FTIR in vivo. Pharm. Dev. Technol. 17, $125-128$

58 Palanisamy, P. (2008) Biosurfactant mediated synthesis of $\mathrm{NiO}$ nanorods. Mater. Lett. 62, 743-746

59 Chen, M.L. et al. (2010) Solution self-assembly and adsorption at the air-water interface of the monorhamnose and dirhamnose rhamnolipids and their mixtures. Langmuir 26, 18281-21829

60 Gang, H.Z. et al. (2011) Molecular dynamics study of surfactin monolayer at the air/water interface. J. Phys. Chem. B 115 , 12770-12777

61 Penfold, J. et al. (2012) Adsorption and self-assembly of biosurfactants studied by neutron reflectivity and small angle neutron scattering: glycolipids, lipopeptides and proteins. Soft Matter 8, 578-591

62 Shen, H.H. et al. (2011) Surfactin structures at interfaces and in solution: the effect of $\mathrm{pH}$ and cations. J. Phys. Chem. B 115, 4427-4435

63 Xie, Y. et al. (2005) Effect of alcohols on the phase behavior of microemulsions formed by a biosurfactant-rhamnolipid. J. Dispers. Sci. Technol. 26, 455-461

64 Helvaci, S.S. et al. (2004) Effect of electrolytes on the surface behavior of rhamnolipids R1 and R2. Colloids Surf. B: Biointerfaces 35, 225-233

65 Han, Y. et al. (2008) Micellization of surfactin and its effect on the aggregate conformation of amyloid $\beta_{1-40}$. J. Phys. Chem. B 112, 15195 15201

66 Malmsten, M. (2006) Soft drug delivery systems. Soft Matter 2, 760 769

67 Nguyen, T.T. and Sabatini, D.A. (2009) Formulating alcohol-free microemulsions using rhamnolipid biosurfactant and rhamnolipid mixtures. J. Surfact. Deterg. 12, 109-115

68 Onaizi, S.A. et al. (2012) Micellization and interfacial behavior of a synthetic surfactant-biosurfactant mixture. Colloids Surf. A 415, 388-393

69 Worakitkanchanakul, W. et al. (2008) Formation of W/O microemulsion based on natural glycolipid biosurfactant. J. Oleo Sci. 57, 55-59

70 Nguyen, T.T. et al. (2010) Biocompatible lecithin-based microemulsions with rhamnolipid and sophorolipid biosurfactants: formulation and potential applications. J. Colloid Interface Sci. 348, 498-504

71 Shim, G.Y. et al. (2009) Cationic surfactin liposomes for enhanced cellular delivery of siRNA. Asian J. Pharm. Sci. 4, 207-214

72 Cheow, W.S. and Hadinoto, K. (2012) Lipid-polymer hybrid nanoparticles with rhamnolipid-triggered release capabilities as anti-biofilm drug delivery vehicles. Particuology 10, 327-333 